\title{
LAS COOPERATIVAS EN EL DESARROLLO AGRARIO DE CHILE
}

\author{
Antonio García Nossa
}

* Borrador para discusión en el Seminario de alto nivel-Las cooperativas en el desarrolllo rural de Chile- Evolución y perspectivas a realizarse en ICIRA entre el 6 y 10 de abril de 1970. Organizado por la Universidad de Chile (CESCO) y el Instituto de Capacitación e Investigación de la Reforma Agraria. Santiago. 1970. 



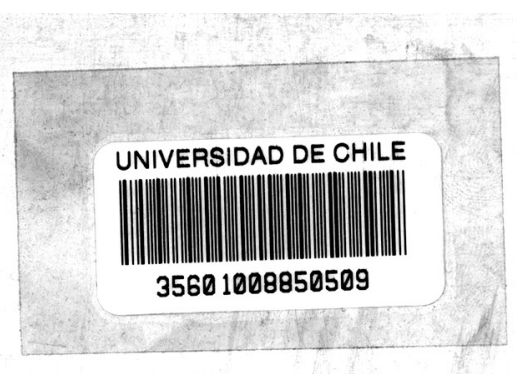

\section{LAS COOPERATIVAS EN EL DESARROLLO}

AGRARIO DE CHILE

ANTONIO GARCIA NOSSA

BORRADOR PARA DISCUSION EN EL SEMINARIO DE ALTO NIVEL - las cooperativas en el desarrollo rural de chile EVOLUCION Y PERSPECTIVAS A REALIZARSE EN ICIRA ENTRE EL 6 Y 10 DE ABRIL DE 1970 ORGANIZADO POR LA UNIVERSIUAD de chile (cesco) y EL INSTITUTO de capacitacion E INVESTIGACION EN REFORMA AGRARIA 


\section{INTRODUCCTON}

Este documento ha sido producto de una larga, intensa y sistemática experiencia de investigación y análisis en las áreas más representativas del campo chileno, experiencia acumulada entre octubre de 1964 y julio de 1968. Durante este periodo - en el cue se constituyó y desarrolló ICIRA como uno de los organismos más valiosos y dinámicos de la América Latina - participé como experto internacional de la FAO y Jefe del Departamento de Ccoperativas, Crédito y Comercialización, dentro de las lineas generajes de orientación trazadas por el Gerente Internacional del Proyecto, Dr. Solon Barraclough y por el Director Nacional, Ing. Antonio Corvalán. Me siento obligado a mencionar la cooperación del primer Director Nacional de ICIRA, Sr. Joacuin Leiva y del Subdirector Ejecutivo,Dr. David Alaluf, asi como del excepcional elenco de expertos internacicnales y chilenos constituido, entre otros, por Almino Affonso, Paulo Freire, Paulo de Tarso, Plinio Sampaio, John Menz, Pedro Moral López, Marcelino Gavilån, Andrew Pearse, Armando Mattelart, Hugo Zemelman, Oscar Dominguez, Adriån Vásquez, Andrés Pascal, Pablo Ramirez, Pedro Hidal go, Enrique Astorga, Juan Carlos Collarte, Bosco Parra, Omar Brevis, Boris Falaka, Jorge Soto, Sergio Gómez, Jorge Mellado, Emilio Klein, etc.

En las investigaciones de campo, en las tabulaciones y análisis de resultados o en los programas de capacitación de ICIRA, participaron activamente mis compañeros de trabajo en el Departamento de Cooperativas, Crédito y Comercialización, Srs. Pedro Hidalgo, Enrioue Astorga Lira, Enrique Contreras y posteriormente Boris Falaha, asi como en los aspectos administrativos y de secretaria la Sra. Maruja Muñoz. Los señores Enricue Astorga y Enrique Contreras (becario del BID) efectuaron investigaciones de campo para estudiar y de- 


\section{IV) sobre financiamiento agricola:}

documentos mimeografiados correspondientes a esas mismas areas.

Demanda de crédito agricola en Chile: áreas de Quillota, Ovalle, Romeral y Rio Bueno.

Estos documentos han servido de base al estudio de evalración critica cue aqui presento, bajo mi entera y exclusiva responsabilidad.

Antonio Garcia

Santiago de Chile, Febrero 12 de 1970 
finir los diversos modelos chilenos de cooperativas agrarias y el Ingeniero Pedro Hidalgo investigó - en esas mismas áreas $\mathrm{y}$ al nivel de los productores campesinos - las condiciones socioeconómicas del financiamiento agrícola, habiendo dirigido, finalmente, el estudio sobre la "demanda del crédito agricola en Chile" en áreas representativas del Valle Central.

Con base en el plan de investigaciones del Departamento de Cooperativas Crédito y Comercialización de ICIRA, se elaboraron los siguientes documentos:

I) sobre cooperativas campesinas:
1) Aconcagua (Mimeógrafo)
2) Marchigüe ( $"$ )
3) Panguipulli ( " )
4) Colchagua (borrador en máçuina)

II) sobre cooperativas agricolas:
1) Llanouihue (Iechera, Mimeógrafo)
2) Caucuenes (Vitivinicola, Mimeógrafo)
3) Elqui (Pisqueras, Mimeógrafo)
4) Aconsagua (Fruticolas, Mimeógrafo)

III) sobre cooperativas tradicionales de colonización y reforma agraria:
1) Choapa (Coquimbo, Mimeógrafo y borrador en máquina)
2) Chiloé (Borrador en máquina)
3) Rio Sur (Llanquihue, Mimeógrafo)
4) Pomuyeto (Ñuble, Mimeógrafo)
5) San Gerardo (Bío-Bioo, Mimeógrafo)
6) Santa Fé ( " " )
7) Lautaro (Cautin, " )
8) Arica (Tarapacâ, " ) 


\section{IAS COOPEFATIVAS EN EL DESARROLLO AGRARTO DE CHIIE}

\section{Antonio Garcia}

\section{PRTMERA PARTE}

\section{LA PERSPECIIVA GTNERAL}

\section{I - Fanorámica de las cooperativas en el sector rural.}

El cooperativismo pural es de formación rcciente y su proceso historico se vincula con las politicas estatales de colonización o de procesamiento industrial de ciertos bienes esenciales de consumo ( como en el caso de la pase. teurización de la leche), con la exigencia económica o con la iniciativa paternalista de medianos y grandes terratenientes (1). Esta forma de constitución de las cooperativas en el sector agropecuario, define las tres grandes lineas socio-eco- nómicas que han adoptado la denominación legal de "cooperativas campesinas", "cooperativas agricolas" y "cooperativas de colonización y reforma agraria". Semejantes denominaciones indican, por si mismas, que el cooperativismo agropecuario no se ha orientado con un sentido funcional de estructura de eambio, sino que ha recogido $\mathrm{y}$ asimilado la estratificación social y económica ( régimen de tenencia, sistema de empresa, estructura y status'social del campesinado ) caracteristica de una estructura agraria de tịpo latifundista/minịfundista. Este hecho central conforma, a grandes rasgos, el tipo de cooperativismo existente en el sector rural de Chile.

En 1939, antes de iniciarse la experiencia de los gobiernos popu-

(1) La Sociedad Hacional de hgricultura tomó diversas iniciativas enderezadas a organizar cooperativas en diversos tramos del sector agropecuario. A estas iniciativas está ligada la dictación de la ley 4331 de 1929 y la orientación de la politica proteccionista del Estado en un sentido de transformar a la cooperativa en un tipo singular de sociedad privilegiada. "El movimiento cooperativo en Chile, Oscar Parrao S., 
listas, se registraban 38 cooperativas agropecuarias, con 1.941 socios (1): el 46\% de los socios pertenecia al estrato alto del cooperstivismo ( el de las llamadas, genéricamente, "agricolas", a más de las fruticolas y lecheras ) y el $40 \%$ al sector de las "Colonias Agrícolas". En un examen objetivo de este proceso hỉstórico, no debe perderse de vista que el esquema de colonización y reforma agraria era de tipo marginal (2), en cuanto no pretendia ni siquiera corregir la estructura lati-

(1) Unión Panamericana, O.C.is, Oficina de Cooperación Agricolia, 1940

(i) Se adopta la clasificación de la reforma agraria en tres grandes tipos:

a) reformas agrarias estructurales, que integran un proceso nacional de transfopmaciones revolucionarias liderizado por el agresivo elenco de nuevas fuerzas sociales de cambio, fundamentándose en la sustitución o alteración profunda de las relaciones tradicionales de poder y en la modificación de sus reglas institucionales;

b) reformas agrarias de tipo convencional que forman parte de una operación entre las antiguas $y$ nuevas fuerzas sociales, por intermedio del sistema institucionalizado de partidos (coiservadores, reformistas y revolucionarios), intentando modificar el monopolio latifundista sobre l. tierra sin cambiar las reglas institucionales de la sociedad tradicional; $\mathrm{y}$

c) reformas acrarias marginales que no apuntan hacia la ruptura del monopolio señorial sobre la tierra o hacia la transformación fundamental de las estructuras latifunuistas (reldciones, poder, sisteme normativo), sino hacia la reparación superficial de esas estructuras, desviando la presión campesina o la presión naciolal sobre la tierra y apoyándose en el sistema tradicional de partidos $\mathrm{y}$ en las reglas institucionales de la sociedad tradicional. Dinámica de las Roformas agrarias en imérica Latina, Antonio Garcia, Santiago,
Edic. ICIRA, 1968. 


\section{$-3-$}

fundista sino más bien inspirarse en sus patrones de innovación tecnológica y social.

En 1964 - un cuarto de siglo después - se registraron 93 cooperativas en el sector (1), con 7.462 socios y una masa financiera constituida por el capital social pagado y reservas al nivel de los 5.7 millones de Escudos corrientes. Las cooperativas del estrato alto ("agricolas" lecheras y vitivinicolas ) representaban el 74 i de las sociedades, con el $76 \%$ de los socios y el $93.25 \%$ del capital pagado més reservas. Un elemento característico de este estrato de medianos y grandes empresarios agricolas, ha sido la contratación de mano de obra ascalariada en la operación normal de la empresa cooperativa: el promedio de obreros contratados (2), ha sido de 14 en las cooperativas agricolas, de 3 en las cooperativas de colonización y de $I$ en las campesinas. El $94 \%$ de los sueldos y salarios pagados a trabajadores contratados por cooperativas, correspondia a sus estratos altos.

Por via de referencie, debe sentarse el notable contraste entre las pequeñas escalas del cooperativismo rural y la amplitud cuantitativa del cooperativismo urbano, proyectando las connotaciones clásicas de la estructura dual. De una masa total de 528.665 socios inscritos en cooperativas con $E^{0} 92.6$ millones de Escudos corrientes de capital social, en 1964, las tres grandes lineas de consumo, ahorro/crédito y vivienda, representaban el $93 \%$ de los socios con el $84 \%$ dél capital social. El cooperativismo de consumo y de vivienda - el más caracteristicamente urbano - agrupaba al $83 \%$ de los socios con el $80 \%$ del capital social.

En 1966, sobre un total de 430 cooperativas con 39.592 socios inscritos, las cooperativas

(1) Ministerio de Economia, Fomento y Reconstrucción, Departamento de Cooperativas. Elaboración ICIRi. 
agricolas constituian el 36\% de las sociedades registradas (1) con el $56.9 \%$ de los socios, las campesinas el. $48.6 \%$ de las sociedades con el 32.9\% de la población afialiada y las de colonización y reforma agr ria el 15.4\% de las cooperativas con el 10\% de los socios. Lún atribuyendo une importancie muy rolativa a este tipo de cuantificación ( ya que se han desconocido datos como los de "sociedades en funcionamiento real", "socios activos", frecuencia de las isambleas Generales, participación democrática, rotación en cargos directivos, etc.), vale la pena senalar la rápida proliferación de las cooperativas campesinas, como efecto probable de las presiones desatades sobre el crédito y los consumos en los más bajos niveles sociales del campo chileno. Sin embargo, este tipo de cooperativismo se ha caracterizado siempro por su marginalismo económico y su indigencia financiera ( capital social promedio - probablemente nada más que susćrito - de 3.000 Escudos corrientes, frente al de $E^{0} 129.000$ de las cooperativas agricolas y de $E^{0}$ 53.000 de las cooperativas de colonización ) (2), con la excepción, reciente, de algunos sectores asistidos o promovidos por INDAP.

En 1967 (3) se registraron 1.477 cooperativas activas con 547.408 socios, correspondiendo al cooperativịsmo de vivienda, consumo y ahorro/crédito, el 65\% de las sociedades en actividad con el $81 \%$ de los socios. Si bien existen cooperativas de consumo $\mathrm{y}$ ahorro/crédito en ciertos sectores rurales ( especialmente en áreas tipicamente latifundistas-minifundistas como las de Aconcagua ), es un tipo de cooperativismo correspondiente a los patrones de la cultura urbana. Las

(1) Servicio de Cooperación Técnica. Se advierte una gran discrepancia con las estadísticas publicadas por 13 Unión Panamericana, correspondientes al periodo 1959/ 1962, en Ios que Chile aparece con 163 cooperativas en el sector agropecuario y 11.500 socios. Ministerio de Economia, Fomento y Reconstrucción, Departamento de Cooperativas.

(2) Servicio de Cooperación Técnica, Encuesta, ob. cit.

(3) Registro de Cooperativas en Septiembre de 1967, Departamento de Cooperativas del Ministerio de Economia, Seminario Latinoamericano de Evaluación sobre Cooperativas, Medellin, 1968. El concepto estadístico de "cooperativas activas" parece limitarse al sentido de que legalmente no han entrado en receso. 


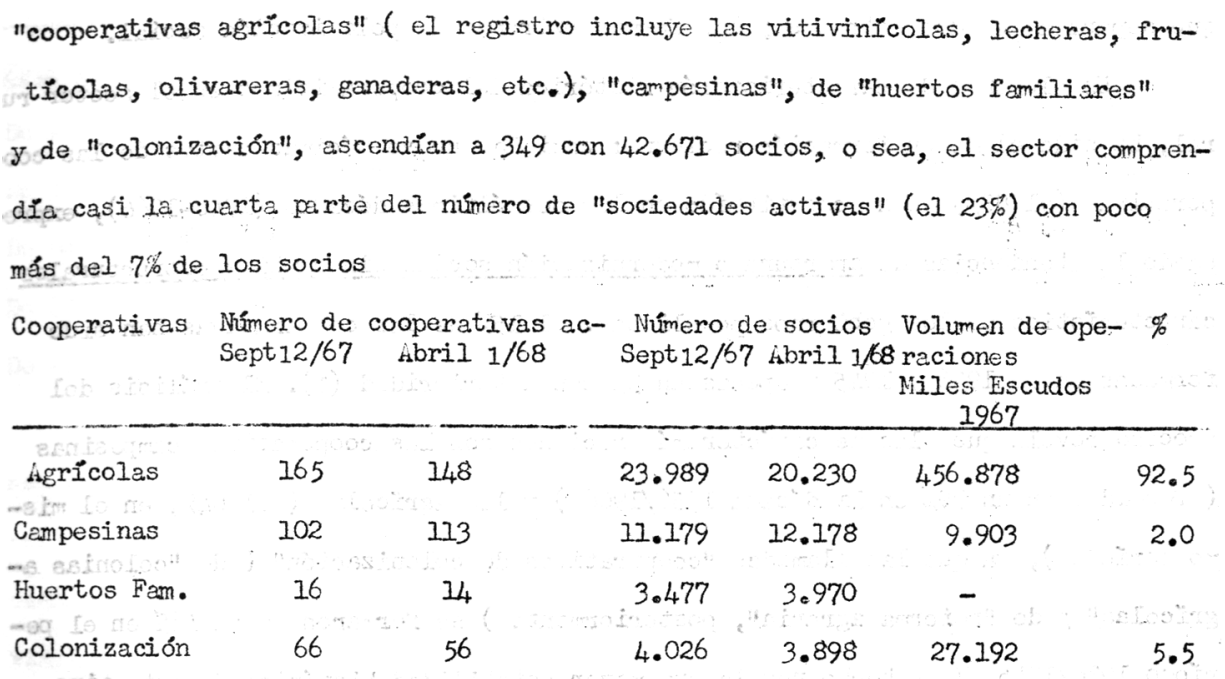

Fuente: Situación del Cooperativismo en Chile, Departamento de Desarrollo Cooperativo; Servicio de Cooperaciór Técnica, Santiago, Mayo 1968.

De las 1.534 cooperativas activas con 529.344 socios, un capital social pagado de $E^{0} 242$ millones $y$ un volumen de operaciones de $E^{0} 1.113$ millones, en Lbril de 1968 (1), corresponde al sector agropecuario el $7 \%$ de los socios, con el $12 \%$ del capital social pagado y el $44.5 \%$ del volumen total de operaciones. El mayor contraste se establece entre el cooperativismo de consumo con el $54 \%$ del número de socios $y$ un volumen de operaciones que sólo llega al 24\% del total, y las "cooperativas agricolis" que con el 3.8\% de los socios, efectian más de las cuatro décimas partes ( $41 \%$ ) del volumen de operaciones. En el sector del cooperativismo rural, las "cooper tivas agricolas" participan en el 93\% del volumen de operaciones y las campesinas apenas en el $2 \%$, projectando la enorme distancia económica

(1) Situación del Cooperativismo en Chile, Departamento de Desarrollo Cooperativo, 1968, ob. cit. 
que separa a los dos estratos y las formas extremas de polarización social.

Un elemento de caracterización histórica del cooperativismo en el sector ru. ral, ha sido el de su formación rociente: casi las nueve décimas partes de las coo. perativas (el 88\%) se ha constituido en las dos últimas décadas (1946-1966), expre. sando las tendencias de apertura o modernización social sin cambios estructurales caracteristico de los gobiemos populistas. El 15\% de las cooperativas han sido formadas entre 1936 y $1945 \mathrm{~J}$ epenas un 3\% con anterioridad (1). El análisis del proceso revela que las de carácter más reciente son las cooperativas campesinas ( formadas en un $99 \%^{\circ}$ en la década 1956/1966) y las agrícolas (el 64\%, en el mismo pcriodo), ya que las Ilamadas "cooperativas de colonización" ( de "colonias agrifcolas" "y de. "roforma agraria", posteriormente) so formaron en un 46\% en el periodo 1936/1945. Este hecho revela una major estabilidad histórica de este tipo formal de cooperativismo ( como se demuestra en el capitiulo pertinente), sino la obligatoriedad de la adhesión por parte de lns asignatarios de tierras y la insignificante magnitud operativa de las organizaciones rurales. Las cifras generales no son enteramente comparables, ya que representan contenidos distintos: mientras las cooperativas agrícolas tienden a constituirse como sociedades de gran tamaño ( asociando poblaciones muy heterogéneas), las cooperativas de colonización o las campesinas son por 10 general de pequeña magnitud $y$ acentuada homogeneidad social ( con excepciones como las correspondientos al esquema de parceleros-huerteros en la reforma agraria de la antigua COR: o de la institución eclesiástica INPROh en Colchagua ).

(1) Servicio de Cooperación Técnica, Departạn ento de Desarrollo Cooperativo, 1967. 
Antiguedad de las cooperativas del sector rural (1)

$\begin{array}{llcr}\text { Escala de edad } & \text { Lgricolas } & \text { Campesinas } & \text { Coloniza } \\ \text { De } 1 \text { a } 2 \text { años } & 15.7 \% & 51.9 \% & 4.5 \\ \text { De } 3 \text { a } 4 \text { años } & 22.4 & 34.3 & 10.6 \\ \text { De más de } 4 \text { a } 12 \text { años } & 27.9 & 12.7 & 18.1 \\ \text { De } 13 \text { a } 22 \text { años } & 17.6 & & 3.0 \\ \text { De más de } 23 \text { años } & 16.3 & & 36.3\end{array}$

Desde el punto de vista de la densidad regional, las mayores concentraciones se efectlian en las Provincias de aconcagua, Valparaíso, Santiago y O'Hïggins, con el 30\% de las cooperativas agropecuarias (2) y en las Provincias de Concepción, irauco, Bío-Bío, Malleco y Cautín, con el 21\%. In el primer grupo de Provincias, la mayor densidad se explica por el auge de las cooperativas agricolas en los altos estritos de este sector del Valle Central ( concentrando el 35\% del total de cooperativas en el pals ), así como por la reciente proljforación de las cooperativas campesinas formades por trobajadores de fundos, precaristas y minifundistas ( con el 35\% de las cooperativas de este tipo en el pars ). En el segundo grupo de Provincias - al sur del Bío-Bío - la densidad se explica por el peso regional tanto de las cooperativas agrícolis, como de las campesinas y las de colonización ( repres ntando el 19\%, el 23\% y el 26\% de los correspondientes tipos en el. país). En este grupo de Provincias se registra la mayor densidad de cooperativas de colonización ( o de "colonias agrícolas", particularmente en Bío-Bío y Cautin ).

Desde el punto de vista de las orientaciones especializadas o pluralistas de la actividad económica, en el cooperativismo del sector rural domina la ten-

(1) Elaboración del Departamento de Cooperativas y Crédito de ICIRis: Total en ictividad, en 1967, 333 cooperativas ( 165 agrícolas, 102 campesinas y 66 de colonización) S. C. T.

(2) Servicio de Cooperación Técnica, CORFO, ob. cit. 
dencia a la multiactividad, perticularmente en el área de las cooperativas campesinas y de colonización. Tanto las grandes cooperativas agrícolas como las ccop rativas campesinas de tipo marginal tienden a la uniactividad, las primeras por una razón de especielización económica y las segundas por carencia de posibilidades de diversificación. En la encuesta del S.C.T. (1967), sólo un 31.6\% de las. cooperetivas operan con una sola actividad económica. Lctividades predominantes en las cooperativas del sector rural (1)

\begin{tabular}{lccc}
\hline & $\begin{array}{c}\text { Agricolas } \\
\text { (Total 130) }\end{array}$ & $\begin{array}{c}\text { P } \text { Cempesinas } \\
\text { (Total 75) }\end{array}$ & $\begin{array}{r}\text { Colonización } \\
\text { (Total 56) }\end{array}$ \\
\hline Abastccimiento Insumos & $61 \%$ & $36 \%$ & $52 \%$ \\
Comercialización Produc. & 57 & 29 & 36 \\
shorro/Crédito & 5.4 & 13.4 & 18 \\
Consumo & 12.3 & 67 & 14.3 \\
Mecanización & 2.3 & 1.3 & 23.2
\end{tabular}

icerca del peso económico de las voperativas, los únicos indicadores disponibles demostrarían la hipótesis de la elevada potencialidad económica de Ias coope rativas lecheras y vitivinicolas ( con el $40.17 \%$ de los socios disponen del $91 \%$ del capital social de las cooperativas del sector ) y el carácter marginal del tipo clésico de cooperativos campesinas ( con el 0.3\% de los socios, operan $010,04 \%$ del cepital social del sector rural ) (1964).

Este fenómeno revela las tendencias características de la estructura latifundista-minifundista: a) la del bajo nivel organizativo del sector rural ( n 1965, la población rural asociada on cooperativas apenas representaba el. 1.4\% de la población actịva del sector ); b) la del alto nivel organizativo de Ios grandas y medianos propietarios de la tierra; y c) la del bajísimo nivel

(1) Servicio de Cooperación Técnica, ob. cit. Pg. 39 - Ios toteles de cooperativas se refieren a las que respondieron la Incuesta. Su.valor és puramente indicativo. 
- organdzativo de los inquilinos, precaristas, minifundistas y trabajadores sin tiewo 11\% Las cooperativas campesinasasociaron, en 1965, al $0.1 \%$ de la población activa del sector, en tanto que las cooperativas lecheras y vitivinicolas agruparon a] $1.5 \%$ de esa población activa. La política de promoción de InDiP ha elevado al 1.5\% y al 7.9\% la proporción de población activa organizada en cooperativas campesinas y en Comités de Pequeños igricultores, respectivamente: en algunas Provincias del Valle Central - como la de Colchagra - esos niveles de organización de la población activa por medio del cooperativismo campesino se han icrementado hasta el $5.3 \%$ (diciembre de 1966 ).

Recientes investigaciones sobre "Ia demanda del crédito agricola en Chile", (2) handefinido más objetivamente esta imagen, al demostrar que la cooperativa es la forma preferente de organizición de las grandes y medianas explotaciones, nó de las pequeñas. En una área eminentomente horticola, como la de Quillota (Provincia de Valparaiso) - en la que presumiblemente domina la pequeña explotación - están organizadas cooperativamente el $\varepsilon 2 \%$ de las grandes explotaciones y el $71 \%$ de las medianas. En las áreas de minifundistas (precaristas o propietarios), predomina el Comité de Pequeños ígricultores y el Sindicato Campesino en el sector de assicriados.

(1) isceptando Ios datos elaborados por la Unión Panamericana para el periodo 1959/ 62 , se registraría para Chile un coeficiente del $1.7 \%$ de población activa agricola afiliada a cooperativas, y para la smérica Latina - excluída irgentina e11.3\%. Argentina exhibía un coeficiente excepcional del $27.3 \%$ de su población activa ( por el peso de las cooperativas de "chacareros" y "tamberos") y el Brasil, un 3.7\%.

(2) Investigación efectuada por el Departamento de 6ooperativas y Crédito de ICIRa, en 1967, en áreas representativas de Ovalle (Coquimbo), Rio Bueno (Valdivia), Romeral (Curicó) y áreas hortícolas de Quillota (Valparaǐso). 
iffiliación a cooperativas, por estratos de productores $1966-1967$

\begin{tabular}{|c|c|c|c|c|c|c|}
\hline $\begin{array}{r}\text { Areas investiga- } \\
\text { das }\end{array}$ & & & $\begin{array}{l}\text { Pequeños } \\
\text { productores }\end{array}$ & $\begin{array}{l}\text { Medianos } \\
\text { productores }\end{array}$ & $\begin{array}{l}\text { Grandes } \\
\text { productores }\end{array}$ & $\begin{array}{l}\text { Total } \\
\text { Muestr }\end{array}$ \\
\hline Ovalle (Coquimbo) & Coop. & Campesinas & $14.3 \%$ & $3.1 \%$ & - & $6.4 \%$ \\
\hline & $"$ & Ligrícolas & - & 31.3 & $98.8 \%$ & \\
\hline $\begin{array}{r}\text { Rio Bueno (VaIdi- } \\
\text { via) }\end{array}$ & $"$ & Campesinas & & & & \\
\hline & $"$ & hgricolas & - & 23.0 & 100 & 41 \\
\hline $\begin{array}{l}\text { Quiliota (Valpa- } \\
\text { raiso) }\end{array}$ & " & $\begin{array}{l}\text { Campesinas } \\
\text { hericolas }\end{array}$ & 22 & 70 & 81.8 & 54.7 \\
\hline
\end{tabular}

A grandes rasgos, podria caracterizarse este tipo de cooperativis mo como una transposición formal del que funciona en los paises desarrollados de Occidente y que se fundamenta en la complementación conómica de la estructura tr dicional de tenencia y ompresa - especialmento en las relaciones activas y pasive con el sistema nacional de mercado $y$ con el cuadro institucional de servicios estatales - y que en modo alguno está diseñado para la promoción o instrumentación de cambios estructurales. De allí que en el cooprativismo dominante en el sector rural, no se advierta ninguna tendencia orientada hacia la promoción de cambios en la estructura de la tenencia, en el sistema de uso de los recursos físicos, en la creación de una nueva infraestructura agricola o en la modificación profunda del esquema tradicional de empresa y de relaciones sociales. Dentro de este marco histórico, es posible comprender el papel del cooperativismo en la instrumentación y propagación de ciertas innovaciones tecnológicas y en la modernizació - racionalización de los procesos de comercialización al nivel de los grupos sociales dominantes en el campo chileno, así como también su incapacidad para alterar el status del campesinado (inquilinos, precaristas, minifundistas, comune- 
neros, peones ), o para desempeñar un papel significativo como estructura de desarrollo económico y social. De alli que las tendencias predominantes en las cooperativas agricolas sean las de captación de servicios asistenciales del Estado o de comercialización (en el sentido más amplio y orgánico del proceso ) y en las cooperativas campesinas de orientación tradicional ( para diferenciarlas de las promovidas y asistidas por INDAP dentro de una nueve concepcion ideológica) sean las tendencias de consumo o de ahorro/crédito, en sustitución o complementación de las antiguas formas organizativas de los fundos clásicos de inquilinaje ("pulpcrías", sistemas de crédito patronal, etc.)

En las masas campesinas no existe ninguna imagon de la cooperativa como sistema de economía y de organización social o se ha formado una imagen distorsionada como pulperia, como institución de préstamos o como forma asociativa de la clase patronal (1).

En las cooperativas agrícolas - integradas a la economia nacional de mercado - un 37\% de la producción es comercializada a traves de la cooperativa y el 30\% de los socios comercializa su producción por intermedio de ella, exclusivamente (SCT).

Esta hipłtesis de trabajo no tiende a negar el activo papel desempeñaLo por este tipo formal de cooperativismo en el proceso de modernización tecnológica y comercial de la agricultura chilena (especialmente en las Inneas lechera, vitivinicola, fruticola, etc. ), sino a definir cuatro fundamentales elemen-

(1) Ver "Lspiraciones de Ios Inquilinos de la Provincia de Santiago, Oscar DomInguez, Santiego de Chile, Edic. ICIRi, 1966. En una investigación efectuada en 25 Comunas de la Hoya del Maule ("El campesino del Maule", Centro de Investigaciones Sociológicas de la Universidad Católica de Chile y Consejo Superior de Fomento sgropecuario, Revista Mensaje, No 147, Santiago, 1966, Pg. 118 ), el 46\% de los campesinos desconocía la utilidad de los sindicatos, el $71 \%$ carecía de concepto sobre la cooperativa y la gran mayoría del $29 \%$ restante la con ebía como una institución de préstamo de herramientas. 
tos de juicio:

a) el primero es el de que se trate de un tipo de cooperativismo compro metico, esencialmente, con la conservación estructural del statu quo ( esquemas de modernización periférica o formal, sin tocar le estructura Istifundista-minifundista ); (1)

b) el segundo es el de quc carece de condiciones o facultades para desempeñar un papel indopendiente, sustancial y dinámico en los modernos proyectos estratégicos de desarrollo nacional;

c) el tercero es el de que no sirve a las comunidades campesinas de colonos, precaristas, minifundistas, comuncros y peones pare movilizar su iniciati interna con un sentido de desarrollo comunal para incorporarlas al sistema nacio nal de mercado y para integrarlas a los mecanismos asiatenciales o representativos del Estado; y

d) el cuarto es el de que es un cooperativismo radicalmente inepto para instrumentar un cambio estructural como la Reforma isgraria.

Es evidente que semejante formulación supone y exige un cambio muy profundo on lo concepción misma del coopcrativismo, ye que de lo que se trata no es de reproducir unos patrones organizativos e ideológicos norteamericanos o europeos, sino de diseñar una estructura que rusponda satisfactoriamente a las exiger cias del descrrollo económico y social de la nación chilena, a las necesidades de orgenización multiactiva del campesinado ( reeducación social, formación empresaria, canalización de servicios asistenciales del Estado ), a la democratización del poder rural $y$ al reto de los cambios estructurales.

Esta es 12 filosofia que inspira el esquema de un cooperativismo concebido como una estructura de desarrollo y de cambio. Su naturaleza, sus alcances, sus tipos, sus métodos operativos, están condicionados por sus funciones on la instrumentación de la reforma agraria estructural. (1) Consultar sobre la problemática de la coopcración on áreas o paises de estructura tradicional. Progress in Iand Reform (1.0 ronnot) nitr ink pares ind 


\section{II - La estructura pública y el cooperativismo del sector rura].}

1. Las instituciones estatales de control, asistencia y fomento

El Estado chileno dispone de un notable repertorio de órganos y recursos destinados a la fiscalización, el fomento y la asistencia técnico-financiera al cooperativismo del sector rural ( posiblemente uno de los más variados y de mayor potencialidad de la américa Latina ), pero semejante cuadro institucional está distorsionado por dos caracteristicas limitentes: la primera es la de que ese repertorio de órganos y recursos institucionales reproduce o proyecta - en el plano superior del Estado - la naturaleza y orientaciores económico sociales del coopcrativismo tradicional; y la segunda es la de que carece de coherencia, articulación $y$ capacidad de actuar unificadamente sobre los diversos tipos de cooperativas en el. sector agropecuario, no existiondo ni un marco de planificación de la acción del Estado ni siquiera un método de coordinación de las políticas desarrolladas en una misma linea operacional. Ni los organismos que operan en el campo del financiamiento - como el Banco del Estado $\mathrm{y}$ CORFO - ni aquellos que desenvuelven una nueva orientación en la política asistencial del Estado - como INDir y CORís - disponen de los más elementales mecanismos de relación o de coordinación de lineas opcracionales. La acción del Istado ( tan fundamental en américa Latina pero mucho más en un pais, como Chile, en el que los sectores estatales controlan cl 70\% de los recursos de inversión nacional ), opera en un senticlo vertical y sectorializado, por medio de órganos estancos $j$ sin relaciones institucionales entre sí, retrasando las posibilidades de que esa acción pública pueda originar un enérgico y equilibrado proceso de cambios. Esta imagen de los órganos públicos corresponde, por lo demás, al de un cooperativismo rural que tiende a asociarse en federaciones de tipo territorial o institutos nacionales destinados a canali- 
zar recursos del Estado, pero que no exhibe tondencias hacia una verdadera integración económica, en la base, por medio del mecanismo de las relaciones intercooperativas. Ni siquiera en regiones de elevada densidad organizativa - como la de sconcagua - funciona este proceso de integración económica al nivel de las estruc turas de región o de área. uńn más: os tal la naturaleza aislacionista del cooperativismo en el sector rur al ( limitándose a la práctica formalista de unos principios de igualdad electoral, adhesión voluntaria y distribución de excedentes ), que ni siquiera las cooperativas o estructuras similares existentes en una misma región y bajo la autoridad de un mismo órgano público ( como ocurre con las colonias $y$ isentamiontos Campesinos en los valles regados de una área desértica, como wica ) están encuadrados dentro de los marcos cohesivos de una plenificación regional. Estos órganos públicos han sido la respuesta, desordenada y sin coherencia, a la demanda de servicios originada en los diversos sectores del campo chiIeno: el que se caracteriza por una agricultura comercial y especializada (cooperativas vitivinicolas y lecheras), el que se encuentra en vias de organización empresaria ( cooperativas de colonias agricolas) o los densos sectores de agricul tura de subsistencia o incipiente relación con la economia de mercado ( cooperativas campesinas).

De otra parte, si bien ha ido conformándose una estructura pública de tipo asistencial - como le representada por INDiP y CORA - no se han definido aún las IIneas básicas operacionales frente a los tres grandes sectores del cooperativismo agropecuario:

a) el primero, el representado por el "cooperativismo agricola" (1), en el que le cooperativa desempeía las funciones de estructura auxiliar de operacion económica del sector privado;

(1) En general, se conscrvan las denominacionos legales para referirse a las diversas clases de cooperativas existentes en Chile. 
b) el segundo, el representado por el "cooperativismo campesino", en el que la cooperativa juega un rol de estructura auxiliar de mejoramiento social o comunitario al nivel de las diversas capas de trabajadores de la tierra; y

c) el tercero, el representado por el "coopcrativismo de colonización y reforma agraria", en el que a la cooperativa se le asigna teóricamente, un papel de estructura básica de cambio y desarrollo económico-social.

Cualquier projecto de planificar el desarrollo de las cooperativas en el sector rural, tendrá que partir de la integración o coordinación de los órganos priblicos ( al nivel del Estado y posiblemente tambión àl nivel de las regiones y de las áreas), asi como del acoplamiento y reajuste de las tres grandes lineas del cooperativismo agropecuario.

2. Iineas operacionales

A grandes rasgos, los órganos públicos relacionados con el sector rural, podrian clasificarse de acuerdo a la naturaleza de sus lineas operacionales, más que por la suma de recursos de que circunstancialmente disponen. II examen funcional de los órganos públicos, permite determinar cinco grandes líntas operacionales:

a) lineas de fiscalización y vigilancia, desde un punto de vista contable y legal;

b) Iineas de nuevo tipo asistencial:

i) asistoncia educacional y técnica;

ii) asistencia financiera;

iii) asistencia de carácter promocional.

c) Iineas de operación financiera de tipo comercial;

d) Iincas de operación comercial:

i) con un sentido asistencial de creación de los mecanismo de nlace con cl sistema nacional de mercado; o 
ii) con un sentido de operación corriente de racionalización del mercadoo agropecuario;

e) Lineas de participación social del Estado en las cooperativas.

\section{a) Linoas de fiscalización y vigi lancia}

In el sentido más estricto, estas funciones se desempeñan en el plano restringido de la vigilancia legal y la fiscalización contable. En consecuencia, su sentido es puramente formal y no tiende ni a orientar, ni a controlar, ni a suministrar asistencia, desde el punto de vista de unas exigencias estructurales.. El órgano operacional es el Departamento de Cooperativas del Ministerio de Economia, Fomento y Reconstrucción, localizado administrativo en un nivel intermedio ( como dependencia - "independiente" - de la Dirección de Industria y Comercio (DIRINCO), con una planta administrativa de 23 funcionarios ( 10 Contadores y 4 labogados ) y una responsabilidad que comprende a la totalidad del cooperativismo chileno, con excepción de las cooperativas de colonización y reforma agraria adscritas a CORh.

EI Depártamento registra, aproximadamente, unas 2.700 cooperativas ( 1.700 con personalidad juridica y 1.000 en tramitación ), lo que supone una disponibilidad funcionaria de 193 cooperativas por profesional. Ia actividad de fiscalización y vigilancia se efectúa, necesariamente, como una operación de gabinete, por medio de la revisión de estatutos, actas y balances periódicos. La desporción entre el tameño del brgano y la dimensión de sus funcionos, determina un largo proceso de tramitación de expedientes de 8, 12 y 18 mescs, no obstante que la personalidad juridica es el requisito indispensable para que las cooperativas puedan entrar en relaciones contractuales con el sector bancario o comercial. En el caso de las cooperativas asistidas por INDiP y como efecto de la intervención de este organismo en la agilización del preceso, se han reducido los 
términos a 2 y 4 meses.

En cuanto a las funciones de revisión de balances, se estima que son rechazados entre un $20 \%$ y un $30 \%$ por falta de requisitos legales ( firma de contador, detalle de cuentas, comprobación de gastos, etc.) Desde luego, el problema se plantea, casi exclusivamente, en las cooperativas campesinas, no sólo en razón del bajo nivel de su capacidad económica, sino de la naturaleza del balance como un elemento de control semejante al que opera en el campo de las sociedades anónimas. El problema es más profundo de lo que a primera vista parece, ya que el objeto del "balance" en las cooperativas no es sólo el de servir de instrumento al control gubernamenial, sino el de operar como elemento del control social, base de sustentación de la gestión democrática. Esta formulación señala la necesidad de que se proyecte un ruevo tipo de contabilidad cooperativa, de acuerdo a sus grandes Iineas, y de que los órganos públicos presten el servicio de asistencia contable, nó en el sentido de confeccionar contabilidades de cooperativas campesinas o de reforma agreria sino de capacitar a las bases sociales en la comprensión y manejo elemental de la cuenta.

Por su misma naturaleza, las funciones de vigilancia y fiscalización d lesempeñadas por COR. en relación a las cooperativas de colonización y reforma agraria, adoptan ciertas formas originales de carácter asistencial.

\section{o) Iinoas de nuevo tipo asistencia. I}

La función asistencial de un órgano público se caracteriza por la tendencia a transferir recursos institucionales con un sentido de promocion de sambios, desendadenando las fuerzas internas de una comunidad campesina o de una iierta área social. Deben diferenciarse, estrictamente, aquellas funciones que son isistenciales, de las que son fomentistas (en el sentido clásico) o aquellas de ipo comercial o caracteristicas de una economia de mercado: De alli que en la caegoría "IInea de nuevo tipo asistencial", se comprendan, exclusivamente, estas 
tres:

i) Ias de asistencia educacional y técnica, que desarrollan INDiP y COR como órganos estatales especializados en la orientación del proceso de la reforma agraria y el desarrollo de la comunidad campesina;

ii) las de asistencia financiera, que también desqrrollan CORA, INDiP y en una cierta medida, el Banco del Estado $\mathrm{Y} \mathrm{EC}_{i_{2}}$; $\mathrm{y}$

iii) las de asistencia de carácter promocional, relacionedas con CORis en la esfera de los asentamientos Campesinosy de las Colonias hgricolas y con INDaP en la do las cooperativas campesinas y los Comités de Pequeños agricultores que constituyen su esfera de influencia.

Uno de los puntos més débiles del cooperativismo radica en la educación cooporativa, al nivel de las comunidades campesinas, ya que por lo general se la ha concobicio dentro de los marcos tradicionales de la cxtensión rural. Los métodos utilizados son casi los mismos empleados por los extensionistas - formaIes, precarios, irregulares, sin capacidad cie desencadenar motivaciones internas en la comunidad campesina - $\mathrm{J}$ on somejante medida desestiman el papel de la masa como agente de cambio. El núcleo de la capacitación parece estar constitujăo por el enunciado de los grandes principios $y$ nó por los problemas que debe afrontar una comunidad campesina pera desarrollarse desde adentro. Es de recordar que cerca de la mitad de los socios de las cooperativas campesines son inquilinos, precaristas u obreros agricolas (1) $\mathrm{T}$ que el 65\% de los socios de las cooperativas agricolas ( de elevada heterogeneidad social) son pequeños propietarios y arrendatarios de tierras.

Tanto CORit como IMDAP se han constiturdo como órganos públicos que no sólo prestan servicios asistenciales de carácter educacional, técnico y financie-

(1) Servicio de Cooperación Técnica, Departamento de Desarrollo Cooperativo, ob. 
ro, sino que tienden a integrar estas formas perticulares de asistencia. En el plano de la capacitación cooperativa al nivel de los funcionarios del Estado, pueden señalarse dos grandes etapas: una, en la que ICIRA ha suministrado una orientación general a los cuadros altos $y$ medios de CORA, INDAP, SAG ( de la que forma parte la enseñanza en el cooperativismo de reforma agraria), la que en 1967 ha Ilegado a comprender 1.500 funcionarios ( 1 ; $y$ una segunda, correspondiente a la Fase II de ICIRA ( ver Conclusiones y Recomendaciones), en la que puede y debe acometer la tarea de proyectar y realizar cursos de capacitación para agentes o promotores de cooperativas de reforma agraria $y$ desarrollo rural. Este plantel de funcionarios ( promotores de cooperativas o agentes de extensión ) podrá enfrentarse a la responsabilidad de la capacitación masiva del campesinado, en las cooperativas de reforma agraria ( bian sean estas asignatarias de tierras o bien asocien a asignatarios de tierras) o en las cooperativas campesinas (desarrollo de la comunidad. rural ).

Las orjginales funciones asjatenciales desempeñadas por ITDAP y CORA, hacen necesario un examen global de sus lineas de opereción cooperativa.

i) Iinea cooperativa de INDAP (cuadro operacional)

INDAP es una Agencia del Istado cuyo papel se relaciona directamente con el desarrollo de las pequeñas comunidades campesinas ( cooperativas campesinas, Comités de Pequeños igricultores, etc.) y es en esta esfera que puede participar en el proceso de la reforma agraria (en cuanto proceso de cambio estructural del campo chileno).

Ia Subdivisión de Asistencia Cooperativa de IMDAP esta compuesta por 41 funcionarios, $29 \%$ de los cuales operan en funciones de asesoria cooperativa y $54 \%$

(1) Ver Informes de la Unidad de Capacitación de ICIRA, Paulo de Tarso Santos. 
en funciones técnicas en el trabajo de campo. Este personal se desempeña bajo la orientación directa de los jefes de zona o de área, y en materia cooperativa depe de,directamente, de la Subdivisión (oficina central), la que forma parte de la Di visión de Campesinos y Pescadores y recibe orientaciones directas del Vicepresidente de INDAP.

Ias cooperativas asistidas ascienden 366 , con 6.755 socios, cerca de 1 a mitad de los cuales se localizan en las Provincias de Santiago, Valparaiso, Cautin y Concepción. EI nưmero de socios por cooperativa es de 102 (promedio nacional ), si bien en algunas Provincias - como en Aconcagua o Coquimbo - la dimensió societaria baja a 78 o 48 socios por cooperativa. En términos nacionales, el núme ro de cooperativas y de socios por asistente es de $3 \mathrm{y}$ de 307. En Provincias de elevada densidad societaria, esa proporción es de 7 cooperativas y 584 socios por asistente en Aconcagua o de 5 cooperativas y 1.235 socios por asistente en O'Higgins, Io que supone un rebasamiento de a capacidad asistencial. El campo de acción de los funcionarios de asistencia no sólo se relaciona con las cooperativas campesinas sino con los grupos pre-cooperativos. La importancia de la estructura asistencial de INDHP - desde el punto de vista de la extensión del área social que cubre - puede estimarse por el hecho de que, sobre 99 cooperativas cam. pesinas con personalidad juridica en 1967 (agosto) - asiste al 66\% de ellas con e $70 \%$ de los socios.

Magnitud del campo asistencial de INDAP

\begin{tabular}{lcccc} 
Estado de las cooperativas & $\begin{array}{l}\text { Ninero de coo- } \% \\
\text { perativas a } \\
\text { sistidas }\end{array}$ & Número de socios & $\%$ \\
\hline $\begin{array}{l}\text { Cooperativas con personali- } \\
\text { dad juridica }\end{array}$ & 66 & $28 \%$ & 6.755 & 38.2 \\
$\begin{array}{l}\text { Cooperativas en proceso de } \\
\text { formación (sin personali- } \\
\text { dad juridica) }\end{array}$ & 171 & 72 & 10.932 & 61.8 \\
Totales & 237 & 17.687 &
\end{tabular}


Esta cifra ( total de socios de cooperativas formadas y en formación ) representaria cerca del $80 \%$ del total de socios del cooperativismo del sector rural, descontando el número correspondiente a los medianos y grandes propietarios (agrupados en las cooperativas agrícolas o en las de colonización)(1)

Desde el punto de vista del estado de las cooperativas en proceso de formación, el $48 \%$ se encuentra en el grado de promoción, el 34\% en la etapa de la organización y el 18\% en estado de legalización.

En cuanto a las 66 cooperativas personalidad jurídica, el examen de una muestra de 54 cooperativas (el $82 \%$ de las sociedades con el $93 \%$ de los socios) permite determinar tanto los tipos de actividad como las diferentes lineas de" servicio:

\begin{tabular}{lcc} 
Tipos de actividad & $\begin{array}{c}\text { Número de lineas de } \\
\text { servicio, en la } \\
\text { muestra }\end{array}$ & $\begin{array}{c}\% \text { de cooperativas } \\
\text { de la muestra }\end{array}$ \\
\hline Consumo & 49 & $44 \%$ \\
Abastecimiento de in- & 29 & 26 \\
Comercialización & 25 & 22 \\
Producción & 9 & 8 \\
\hline T o t a I & 112 & 100
\end{tabular}

El análisịs de estos resultados permiti adoptar las siguientes conclusiones:

a) en promedio, cualquier cooperativa tiene más de dos lineas de servicio;

b) el $44 \%$ de los servicios corresponden a la linea del consumo; y

c) casi la totalidad de actividades (el 92\%) forma parte del sistema de relación externa de una comunidad campesina con la economía de mercado. Sin embargo, la clasificación más importante - desde el punto de vista social - es la de cooperativa uniactivas y cooperativas multiactivas: las primeras son aquellas que

(1) Servicio de Cooperación Técnica, Departamento de Desarrollo Cooperativo, Encuesta a Cooperativas del sector hgropecuario, ob. cit. 
están asociadas a una sola actividad ( casi exclusivamente el consumo), no por razones económicas de especialización ( como en el caso de las cooperativas leche ras o vitivinicolas) sino de niveles próximos a la miseria ( esta es la situación del $41 \%$ de las cooperativas con el $38 \%$ de los socios ); las cooperativas multiactivas son aquellas que tienen dos lineas de actividad ( el 35\% de las cooperativa con el $34 \%$ de los socios ) o más de dos lineas, como ocurre con una cuarta parte de las cooperativas de la muestra con el $28 \%$ de los socjos. La multiactividad puede expresar - como en el caso de las comunidades campesinas en las áreas de latifundio/minifundio de Aconcagua - una presión múltiple de necesidades originadas en la explotación, las relaciones con el mercado o en la economia familiar, - simplemente puede ser el producto de cierta asociación natural de actividades, como ocurre con la comercialización agropecuaria y la obtención de insumos agrícolas o bienes de uso familiar. Es significativo él hecho de que ni la tercera parte de los socios pertenece a cooperativas multiactivas, no obstante el papel de la cooperativa como estructura de canalización de servicio asistenciales de INDAP.

La asistencia financiera de INDAP ( cuyos resultados se analizan en el proceso de las cooperativaścampesinas), adopta la doble tendencia del crédito de capitalización ( a cooperativas 0 a productores) y del crédito operacional. Al parecer, la linea básica se orienta en el sentido de financiamiento de los servicios cooperativos.

En los tres años últimos ( 1965,1966 y 1967) (1), la asistencia financiera cubrió a 17 cooperativas ( con 19 operaciones de crédito), con montos promedio por cooperativa que ascendierop,progresivamente, de $E^{0} 14.843$ en $1965, \ldots$ $E^{0} 22.800$ en 1966 a E0 57.729 en 1967 ( 8 meses) (Escudos corrientes). En términos

(1) 1967 comprende hasta el 30 de Agosto. 
generales, debe observarse que de las 66 cooperativas con personalidad juridica que atiende INDAP, sólo el $16.6 \%$ recibieron crédito ( hasta agosto de 1967 ) y que una sola cooperativa ( la de Marchigüe, en Colchagua ) recibió el 57.8\% de los préstamos a cooperativas en dicho año.

\section{ii) Innea cooperativa de CORi (cuadro operacional)}

CORH es el órgano estatal de ejecución de la reforma agraria $y$ en consecuencia, sus relaciones se limitan a tres grandes esferas de actividad cooperativa: la de los Asentamientos, que pese a su condición transitoria, funcionan dentro de un régimen de sociedad con el Estado y operan con difcruntes métodos comunitarios o cooperativos informales; la de las Cooperativas de Colonias hgrícolas, resultantes de operaciones de redistribución de fundos de inquilinaje ( añtigua reforma agraria ); y la de cooperativas que estrictamente deben llamarsé de colonización porque operan en base a la adjudicación de tierras baldias fiscales, como ocurre en la región Magallánica. En el lenguaje corriente, la denominación de Acooperativas de colonización" sirve para designar los dos tipos últimos.

Desde 1964 desapareció el Departamento de Cooperativas de CORA y fué transformado en una Sección del Departamento de Desarrollo Campesino, indicando con semejante localización administrativa que no se asigna al cooperativismo una función autónoma y sustantiva dentro del proceso de reforma agraria, sino un papel que aún está por definirse, lo mismo que el esquema organizativo del Asentamiento y el de post-ksentamiento. De acuerdo a este concepto, la planta administrativa de la Sección de Cooperativas de CORA es de 4 técnicos, incluyendo al jefe de la Sección. Sin embargo, la actividad cooperativa informal de CORA se realiza a través de los funcionarios del Departamento de Desarrollo Campesino y por medio de la promoción de Comités de Consumo on los isentamientos. Por su misma naturaleza, un Comité de Consumo se constituye como un grupo cooperativo informal, que opcra tano en el abastecimiento de bienes de consumo familiar 


$$
-21
$$

como en la lines de los insumos agrícolss. De otre parte, la constitución democrática de los Consejos de Administración, la explotación agricola por medio de equipos o grupos de trabajo del isentamiento y la distribución de los ingresos de acuerdo a los aportes de trabajo, conforman un tipo de actividad cooperativa aún cuando no hayan adquirido un carácter absolutamente formal.

La capacidad asistencial de CORA se orienta en dos grandes lineas: la de un tipo de abistencia técnicominanciera a los isentamientos Campesinos, dentro del marco del contrato de sociedad; y la de suministro de créditos a las cooperativas de colonización, bien en la forma de préstamos directos o de movilización de recursos originados en diversas fuentes institucionales. En 1965, de un total de 5.3 millones de Escudos movilizados por medio de las dos vias, el $57 \%$ corresponde a préstamos directos y el 43\% a otras fuentes, con el aval de CORA. En 1966, la proporción de los préstamos directos se eleva al 61\% (1). Dos peculiaridades pueden observarse en relación a la line a de movilización de recursos financieros originados en diversas fuentes institucionales: la primera es la de que estos recursos provienen, un cerca de nueve décimas partes, del Banco del Estado; y la segunda es la de que se conservan las formas de concentración características de la distribución comercial del crédito agricola ( una sola cooperativa obtiené el $80 \%$ de los préstamós bancarios ). En 1966, el 97\% de los "créditos indirectos" corresponde a 3 cooperativas, por medio de la utilización de tres fuentes institucionales de crédito: el Banco del Estado, con.el 84.9\%; ECA, con el 12\%; y CORFO, con el $31 \%$ de los recursos movilizados. En este mismo año, los "parceleros" beneficiados con crédito solo fueron 1.062 ( con montos promedio de $\mathbb{E}^{0} 3.643$ ) siendo insignificantes los préstcmos a "huerteros" ( peones con tierra, dentro

(1) Fuente: Departamento de Producción, Sección de Créditos, CoRh, hgosto de 1967 
del antiguo esquema de reforma agraria).

iii) Iinea cooperativa deI Servicio igriocola y Ganadero - ShG del Ministerio de fericultura (cuadro operacional)

Ia Sección de Cooperativas del SAG se caracteriza por estar encuadrada dentro del esquema extensionista tradicional y por orientar su actividad asistencial, exclusivamente, hacia las "cooperativas agricolas". De acuerdo a estos objetivos funcionales, la Sección forma parte del Departamento de Extensión del Servicio Agricola y Ganadero, dispone de una planta administrativa de 6 Ingenieros kgrónomos y 5 Prácticos hgriccolas y desempeña funciones de capacitación cooperativa, promoción y organización. En términos nacionales, su campo de operación está compuesto por 25 cooperativas con 879 socios, 10 que arroja un promedio de 35 socios por cooperativa y 2 cooperativas con 80 socios por cada agente extensionista. La mitad de estas cooperativas se localiza en las Provincias de Santiago, Valparaíso y Nuble.

Ia asistencia técnica versa sobre "estudios de mercado, asesoria en planes de explotación o en aspectos contables y legales", pero sólo aparecen cubiertas 14 cooperativas con 539 socios ( 10 cooperativas agrícolas y 2 campesinas ), esto es, un tramo muy reducido del cooperativismo en el sector agropecuario.

c) Iineas de operación financiera de tipo comercial

La operación financiera de tipo comercial es aqueila que se realiza por instituciones públicas como el Banco del Estado, CORFO y ECA, siguiendo las normas corrientes en el mercado del cridito agricola. De alli que, pesc a ciertas modalidades del crédito que suministra a las cooperativas el Banco del Estado, se le incluya dentro de la categoría del crédito de tipo comercial. De otra parte, las cooperativas con acceso a esta clase de recursos, son, exclusivamente, aquellas que por su solidez financiera y su sistema de garantias pueden equipararse a las sociedades de capital. 


\section{i) Innea de operación del Banco del Estado ( cuadro operacional)}

La Sección de Cooperativas del Banco del Fsstado dispone de una plante ad, ministrativa de 11 funcionarios ( 2 Contadores y 9 asesores ), destinados a veri, ficar la operabilidad comercial o solvencia económica de las cooperativas que so. licitan recursos de crédito. Desde el punto de vista del nivel administrativo, la sección es un dependencia de la Subgerencia de Promoción y hhorro. En última instancia, las funciones de la agencia son simplemente inspectoras y asesoras de una Subgerencia bancaria. No existiendo un tipo de crédito especializado a las cooperativas, el Banco opera dentro de los marcos del régimen ordinario de préstamos y descuentos. De alli que, on 1967 (agosto), corca de las nueve décimas partes de la cartera se hubiese destinado a cooperativas agricolas y apenas el 0.2\% a cooperativas campesinas. Una de las razonos de esta forma de distribución de los recursos financieros es le de que el Banco del Estado opera un tipo de "crédito agrícola" que es nada más qu una modalidad contable del crédito comercial ( corto plazo y tasas de interés ) y de allí que el 92\% de los descuentos se destinen a las cooperativas agricolas. El monto promedio de los préstamos, por tipo de cooperativa, revela también el impacto que este tipo de crédito puede tener en el cooperativismo del sector:

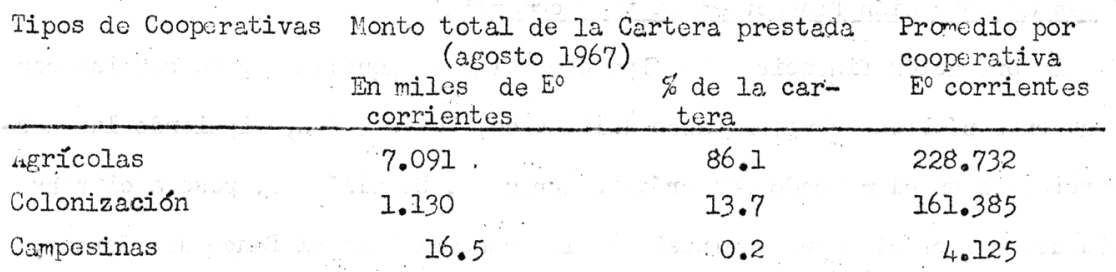

La característica comercial de esta variable del "erédito agrícola", explica el que la distribución siga las mismas tendencias de concentración caracterÍsticas del mercado bancario: en 1966; se movilizaron recursos hacia las coo- 
perativas agricolas por valor de $E^{0} 10.4$ millones ( préstamos y descuentos del Banco del Estado) correspondiendo a 6 cooperativas el $72 \%$ de la masa total de créditos.

\section{ii) Iínea de operación de CORFO ( cuadro operacional)}

Ia CORFO carece de un órgano especializado en el financiamiento de las cooperativas, no obstante que se trata de la más importante estructura pública. de movilización de recursos financieros a largo plazo y que opera dentro de ciertas normas de elaboración racional de proyectos. Fn consecuencia, las operaciones de crédito a las cooperativas se canalizan a través de los Departamentos de Crédito, de hgricultura, de Desarrollo Ganadero y de Operación. Ias exigencias para el financiamiento suponen una operación exclusiva de CORFO con las cooperativas que asocian a los grandes y medianos empresarios agrícolas, estimulando el proceso de "causación circular acumulativá" (G. Myrdal) que consiste en que las pequeñas cooperativas rurales no pueden capitalizarse utilizando recursos de crédito, porque están descapitalizadas; y el crédito de capitalización a largo plazo se orienta hacia las cooperativas de grandes propietarios o empresarios, porque son cooperativas appitalizadas. De alli que, no obstante la elevada movilización de recursos financieros efectuada por medio de CORFO, sea en extremo reducida su frea social de influencia y el impacto sobre el desarrollo rural. En 1960, CORFO prestó a 2 cooperativas por cerca de $E^{0} 100.000$ y en 1966 se habia elevado a 21 el número de sociedades con un monto de cèrca de 11 millones de Escudos corrientes. En los últimos cinco años (1963/1967), fluctó entre 13 y 21 el número de cooperativas financiadas, con montos promedio, por cooperativa, en proceso ascendente: de $\mathrm{E}^{0} 100.330$ en 1964 a más de medio millón de Escudos corrientes en los dos últimos años. Dada la orientación comercial de ln CORFO, se explica el que casi la mitad de su cartera $(45.6 \%$, en 1966) se huoiese orientado hacia las 
cooperativas vitivinicolas ( ampliación de capacidad instalada) y el que, no ob tante el limitadisimo campo de operación, hubiese destinado, en los últimos años una proporción creciento de sus disponibilidades a este tipo de crédito ( $4 \%$ en 1964 a $13.4 \%$ en 1966 ). En 1966, $166 \%$ del monto de los préstamos se destinó a cooperativas lecheras y vitivinicolas ( con promedio de $E^{0} 332.500$ y $E^{0} 855.330$ po cooperativa, respectivamente) y un tercio de las cooperativas - siguiendo las I neas generales de la concentración del crédito en el campo chileno - obtuvo el 7 de los recursos financieros. No obstante la naturales pública de la CORFO y la in sistencia en los procesos de colonización y reforma agraria, la destinación de $\mathbf{m}$ cursos a las cooperativas de este sector apenas representó el $0.5 \%$ del monto de los préstamos efectuados y un promedio por cooperativa de E0 19.180 (1966).

\section{iii) Iinea de operación de $\mathrm{EC}_{n}$}

ECh es un órgano del Estado que no sólo desarrolla funciones de comercializa ción agropecuaria, sino de financiamiento agricola dentro de ciertas pautas comel ciales. De otra parte, la actividad que reaiza no es exclusivamente operacional, yq que se ha vinculado con dos tipos modernos de procesos: el de la promoción cor perrativa $y$ el de las relaciones con los isent mientos Campesinos. Como instituci promocional, ha proyectado y formado a IisUCOOP; mecanismo de distribución coopes tiva de 50.000 toneladas de superfosfatos adquiridos mediante un préstamo de la LIID por US \$3.6 millones. La distribución de estos créditos en especie, a largo plazo, entre 46 cooperativas agricolas y utilizando el mecanismo de las letras ( en favor de las cooperativas, endosadas a la orden de $\mathrm{ECH}_{\mathrm{i}}$ en el campo del fina ciamiento agricolas. De otra parte, las relaciones contractuales establecidas col CORa para la comercialización de ciertas lineas de los tisentamientos Campesinos - como las horticolas y de chacareria - ha abierto la doble posibilidad d que la estructuras oricinadas en la reforma agraria puedan encontrar 
una adecuada vǐa de comercialización agropecuaria y de que las comunidades campesinas ligadas a esas estructuras puedan integrarse, desde el primer ciclo de la reforma, al sistoma nacional de mercado.

Dentro de esta perspectiva, ECh pxdrá definir cuatro grandes lineas de operación en la esfera de las cooperativas del sector rural:

a) la de comercialización agropecuiria en relación a los diferentes tipos de cooperativas tradicionales y operando como el más importante mecanismo de enlace con el sistoma nacional de mercado;

b) la de promoción y asistencia técnica al cooperativismo vinculadoal proceso de reforma agraria (planes y normas de comercializacion );

c) la del financiamiento a cooperativas de diferente tipo, por medio del manejo de créditos externos on insumos, bienes de capital, etc.; $\mathrm{y}$

d) la del abastecimiento en diferentes clases do bienes a las cooperativas. ECA desempeña estas funciones por medio de una Subgerencia de Cooperativas, con une planta administrativa de 8 funcionsios ( 2 Contadores, 3 Agrónomos y 3 iuxiliares administrativos). Ia Subgerencia funciona como órgeno asesor de ln Vicepresidencia y depende jerárquicamente de la Gerencia General. En consecuencia, no opera con las cooperativas directamente sino por medio de los órganos ordinarios de la. institución.

d) Iincas de operación comercial

anteriormente se ha diseñado el cuadro funcional de $\mathrm{ECA}$, como estructura pública de comercialización $y$ de financiamiento.

El desarrollo del proceso de reforma agraria, ha de plantear la necesidad de transformar a ECA en el mecanismo por excelencia de enlace - para los Asentamientos Campesinos o las cooperativas que los sustituyen - con ol sistema nacional de mercado. Desde este punto de vista, tendrá que definirse un sistema contractual 
de relaciones entre ECA y el cooperativismo de reforma agraria. In la medida en que pueda desempeñar estas funciones, ECA estará también opcrando como un enérgis mecanismo de racionalización del mercadeo agropecuario, orientado hacia la export ción o hacia el mercado interno.

Este cuadro de posibiliciades, exigiré una diferenciación, muy estricta, , tre les funciones normales de comercialización agropecuaria y los servicios de ci rácter asistencial, on relación con las nuevas estructuras de cambio. e) Iineas de participación social del Estado en las cooperativas

Un hecho notable del cooperativismo de colunización y reforma agraria (4, Chile o en otros países latinoamericanos como México, Bolivia, Uruguay) es el df que el Estado ha entrado a participar on él por medio de inversiones y transferel cia masiva de recursos, nó sólo por las viaas institucionales corrientes sino por medio de relaciones de sociedad. Dentro de este marco, el Estado opera como dos coses distintas: como aparato político y como socio. La "participeción social" del Estado en las cooperativas conforma un sistema original, en cuanto sus aportes, transferencias o comportamiento se regulan, nó por los principios generales del Derecho Público o del Derecho Privado, sino por las normas específicas del cooperativismo.

La influencia ideolófica del cooperativismo norteamericano y europeo (el cuanto se fundamente on el "horror al Estado" o en el repudio a cualquier forma 1 asociación entre Estado y cooperativas ), ha impedido comprender los alcances, de sarrollar la teorla y lograr la institucionalización de ese tipo peculiar de "cor perativas de participación estatal". En el capitulo final se analizará el posibl campo de operación y la posible estructura de la cooperativa de participación es. tatal, dentro del contexto de una tipologia de coopcrativas latinoamericanas de reforma agraria. 
En el caso de Chile, la constitución del hsentamiento como una sociedad de trabajo entre el Estado (CORia) y una comunidad campesina, asi como la posibilidad legal de que la cooperativa sea asignataria de tierras, atribuye especial significación a este tipo de cooperativismo de participación estatal, especialmente en las árcas en las que se ha ef'ectuado una cuantiosa inversión pública o en las que las cooperativas pueden jugar un papel decisivo en la planificación del desarrollo regional como ocurre en los valles nortinos de trica o en los del Choapa.

\section{III - Conclusiones generales sobre la Estructura Pública}

Este examen suscinto de la estructura pública chilena, ha permitido reconocer el cuadro operacional de siete organismos bésicos relacionedos directamente con cl cooperativismo del sector agropecuario, con un sentido de asistencia, fonento, fiscalización contable, vigilancia legal u operación comercial y financiera. 


\begin{tabular}{|c|c|c|c|c|}
\hline Instituciones & Profesionales* & Técnicos $* *$ & Personal Luxiliar & Total $* * *$ \\
\hline INDLP & 3 & 31 & 7 & 41 \\
\hline COR.Ai & 4 & - & 1 & 5 \\
\hline Ministerio Economi゙ & 14 & - & 9 & 23 \\
\hline$S_{H G}$ & 6 & 5 & 2 & 13 \\
\hline Banco del Estado & 2 & 7 & 2 & 11 \\
\hline CORFO & - & - & - & $\mathrm{s} / \mathrm{d}$ \\
\hline $\mathrm{EC} \mathrm{C}_{\text {A }}$ & 5 & - & 3 & -8 \\
\hline$I \circ t a I$ & 34 & 43 & 24 & 101 \\
\hline
\end{tabular}

(*) Profesionales universitarios y contadores

* Compronde niveles medios, como prácticos agricolas

**\% Debe recordarse que este no es sino ul personal que opura en agencias especializadas on las relaciones con las cooperativas del sector rural y que, en casos como el del Minișterio de Lconomía, el Departamento de Cooperativas dispone de funcionarios destinados a vigilar y fiscalizar el cooperativismo de todos los sectores.

El reconocimiento de este cuadro institucional de órganos del Estado, hace posible señalar algunas conclusiones de caracterización general:

a) se han multiplicado notablemente los órganos públicos de relación con el cooperativismo agropecuario, sin que se haya definido un sistema de rolaciones, ni se haya logrado una especialización funcional;

b) los órganos públicos se han ajustado más a las estructuras administrativas a que pertenecen que a las condiciones y problumas de los tramos cooperativos que cubren en el sector rural;

c) no ha existido una integración o una linca de coordinación operacional entre los diversos órganos públicos, ni siquiera entre aquellos que están inspirados on una semejante filosofía asistencial;

d) no obstante la notable disponibilidad de recursos técnico-financieros 
ən ciertos órganos públicos, la acción general del Estado aparece como insuficiente y débil por la carencia de una planificación nacional del desarrollo del cooperativismo agropecuario, fundementada en la coordinación y en el encuădramiento operacional de aquellos órganos (asignación planificada de recursos, acción por medio de órganos especializados, intercambio de experiencias, etc.);

e) Los órganos públicos han carecido, en general, de jerarquía administrativa, siendo locnlizados en niveles medios o ba.jos y sin autonomia operacional ( con excepcionos como la del Departamento de Cooperativas del Ministerio de Economia en relación con las funcionas de vigilancia logal y de fiscalización contable );

f) no ha existido una filosofĩa o una doctrina común que inspire la acción de los diferentes órganos públicos;

g) Is concentración de recursos públicos y servicios asistenciales en ciertos tramos del cooperativismo rural - especialmente el de las cooperativas agricolas de grandes y medianos empresarios - ha acontuado la estratificación social de las cooperativas y retrasado el proceso de integración;

h) la capacitación cooperativa se ha limitado a la propagación formal de ciertos esquemas clásicos, sin enfrentar la problemática de creación de una nueva estructura de organización empresaria, de participación democratica, de control social, de planificación y desarrollo interno de la comunidad campesina;

i) se ha enfocado el problema de la integración cooperativa desde el punto de vista de los intereses de la economía privada y nó desde el punto de vista de la posibilidad de construir un sector independiente de relaciones intercooperativas y un tipo de. desarrollo autosostenido.

Estc cuadro de órganos y de formas de operación del Estado no puede juzgarse desde una perspectiva de racionalidad formal, si se atribuye al cooperati- 
vismo - y particularmente al de colonización y reforma agraria - un papel sustantivo y activo en el proceso de cambios estructuralos. Por lo mismo que la reforma agraria no es una limitada operación de modernización formal de la emprese o de simple mejoramiento de la comunidad rural, el cooperativismo que la instrumente debe adocuarse a la naturaleza, objetivos y celeridad del cambio. La reforma agraria y las necesidades estretégicas de un cambio estructural on Chilo $\mathrm{y}$ en América Latina, plantean la nocesidad de un nuevo enfocue del coo perativismo y de les estructuras de cambio social. Eso nuevo enfoque debe consi tir en la redofinición de su papel, de sus tipos, de sus formas operativas, des. de el punto de vista de las condicionos y problomas de la comunidad campesina on el proceso de reforma agraria y de los recursos movilizabies por medio de órganos directos o descentralizados del Estado. Las premisas o requisitos de apertura al nuevo proceso, podrían esquematizarse así:

a) Planificación de 13 distribuci in de recursos técnico-financieros del Estado al cooperativismo del sector rural, por medio de un Plan indicativo único que señale normas, tipos de recursos, montos probables, plazos y formas de recuperabilidad, etc., a corto, mediano y largo plazo, para las cooperativas de reforma agraria y cambio social ( una cierta proporción de las coope rativas campesinas y de las agricolas, de acuerdo a la tipificación establecida en el capituio fina ) y a corto plazo para las cooperativas agrïcolas de grandes y medianos empresarios;

b) Readecuación o reestructuración de los órganos públicos que operan directemente con el cooperativismo del sector rural, on el triple sentido de modificar su organización y sus técnicas operativas, de redefinir sus campos de trabajo y de establecer un sistema de coordineción, de planificación y de consulta; 
c) Planificación del desarrollo del cooperativismo de reforma agraria y cambio social, por medio de estructuras coopcrativas regionales de segundo grado (áreas y regiones).

\section{IV - Procesos y niveles de integración del cooperativismo rural}

Pese a la pluralidad de órganos estatales de promoción, regulación y asistencia técnico-financiera y al auge económico de cierto tipo especializado de cooperativas como las vitivinicolas y las licheras, ol cooperativismo del sector agropecuario tiende a desarrollarse en forma de compartimentos estancos. Este tipo de desarrollo confirma la hipótesis de que la cooperativa campesina carece de fuerzasimpulsoras que estimulen la integración - al nivel de las masas rurales, de las regiones y del sistema nacional de mercado - y de que ia "cooperativa agricola" tiende a operar dentro de los marcos normativos del sistema de economia privada. Iy a funcionar como un mecanismo de captaci in de recursos asistenciales del Estado al nivel de los grandes y medianos propietarios. Las tendencias a la integración no se orientan hacia la creación de un sistema de relaciones intercooperativas, sino hacia la formación de aparatos formales o hacia la instalación de ese tipo de estructuras secundarias adecuadas a la inclinación capitalista del sistema de empresa agropecuaria. Ni siquiera en áreas de latifundio/minifundio en las que se ha desviado la presión campesina sobre la tierra por medio de un indigente cooperativismo de consumo o de ahorro/crédito, la proliferación de organizaciones socialos no ha servido para estimular la formación de cooperativas de segundo grado o el establecimiento de un sistema de relaciones intercoopera- 
tivas (1).

Las cooperativas de consumo en esas áreas de latifundio/minifundio pueden i clinarse hacia la constitución de Fëderaciones Territorialos de valor simplement representativo, pero nó hacia la formación de una Central Cooperativa de Compras Las coopcrativas de las "colonias agrícolas" constituidas en una región tan atra da como la Isla de Chiloé ( en la que operan todos los servicios asistenciales d Estado sobre una semejante área social), no se han propuesto siquiera la posibi dad de la integración al nivel insular, no obstante que seria esta la condición ritaria para planificar o coordinar la distribución do recursos públicos de asis cia técnico-financiera (INDhP, CORA, CORFO, ECA, Banco del Estado, etc.) al nive: de las explotaciones campesinas.

Ia coordinación de los órganos públicos no sblo a nivel nacional de los plas Indicativos sino a nivel de las regiones o áreas donue han de ejecutarse, es la dición no sólo primera sino previa a cualquier propósito de planificación del des rrollo.

Sin embargo, existon algunos tipos de experiencias de integración que vale : pena enunciar, a grandes rasgos:

1) el de las Sociedades Auxiliares o el de cierta clase de "Cooperativas $\mathrm{Na}$ cionales";

2) el de las relacionos intercooperativas de comercialización;

3) el de las Federaciones o Uniones Territoriales; y

4) el de las relaciones institucionales entre el cooperativismo y el sector de la economia estatal.

Desde luego, este examen del proceso y de los niveles de la integración coop rativa en el campo chilcno, no se refieren a la problemática concreta de la integración al nivel de las cooperativas de reforma agraria, ni intentan otra cosa qu lograr el diseño de un marco general.

(1) En Ia Comuna de San Esteban, en iconcagua, funcionan 83 diferentes organizaci zaciones, incluyendo 3 cooperativas: sin embargo en una Provincia de acentuada porlarización de la tenencia de la tierra ( el 1.5\% de las explotaciones - con más de 200 hectéreas posee del $94.4 \%$ de la superficie agrícola y el 76\%, con menos de 5 hectáreas, apenas dispone del 1.3\%, de acuerdo a los últimos datos censales), no existe una sola estructura de integración capaz de articular el esfuerzo de las comunidades campesinas y de operar como una fuerza de presión de la reforma agraria. Cooperativas y financiamiento agrícola en hconcagua, Ed: ICIR $x_{2}$, Departamento de Cooperativas y Crédito, 1968, Pg. 134. 